\title{
Evidence-Based Practice: proposal for an extended definition
}

Adrien Pallot, PT, MSc ${ }^{1,2}$, Matthieu Guémann, PT, PhD ${ }^{3}$, Aurélie Morichon, PT, MSc ${ }^{1,2,4}$, Sébastien Martin, PT, MSc ${ }^{4}$, Marion Gallois, PT, MSc ${ }^{5}$, Guillaume Raynal, PT, MSc ${ }^{1}$, Thomas Davergne, PT, PhD ${ }^{1,6}$, Rostagno Stephan, PT, MSc ${ }^{8}$ and Thomas Osinski, PT, $\mathbf{P h D}^{1,2,7}$

${ }^{1}$ CEERRF, Saint-Denis, France, ${ }^{2}$ Université de Picardie Jules Verne UFR de Médecine, Institut d'Ingénierie de la Santé, Amiens, France, ${ }^{3}$ Institut de Recherche Biomédicale des Armées Unité de Physiologie de l'Exercice et des Activités en Conditions Extrêmes, Brétigny-sur-Orge, France , ${ }^{4} \mathrm{EFOM}$, Paris, France, ${ }^{5} \mathrm{CHRO}$,Orléans, France, ${ }^{6}$ Institut Pierre Louis d'Epidémiologie et de Santé Publique, Paris, France, ${ }^{7}$ Unité de Recherche UR 20201 ERPHAN, Université Versailles Saint Quentin, France

DOI: $10.52057 /$ erj.v2i1.3

received: 21 May 2021

accepted: 20 August 2021

\section{ABSTRACT}

Evidence-Based Practice (EBP) is an approach that has emerged in medical practice. In 2000, Sackett et al. stated the most common definition of the EBP approach: "the integration of the best research evidence with clinical expertise and patient values". Over time, many elements have been added and discussed by various authors to this initial definition (cost and duration of the intervention, therapist skills, regional resources provided for health, cultural influences, ...). With evolution of the EBP approach, and these successive additions, we wish to propose a new definition of the EBP in order to bring it a more contemporary and complete face. For that reason, the different objectives of this article are:

- to explain why making such a proposal: a new extended definition

- to propose a new extended definition

- to explain our semantic choices

- to propose (and explain) an updated graphic model for the extended definition

The definition we have adopted is that EBP is the reasoned, individualized clinical application of the highest level of evidence possible in a unique multidimensional context of care.

KEYWORDS: clinical reasoning, decision making process, evidence-based practice, patient-centered care

Translated from Pallot A, Davergne T, Gallois M, Guémann M, Martin S, Morichon A, Osinski T, Raynal G, Rostagno S. Démarche EBP. Evidence-based practice en rééducation - Démarche pour une pratique raisonnée (2019): 11-16. 2019 Elsevier Masson SAS.

Corresponding author:

Adrien Pallot, CEERRF, 36 rue Pinel, 93200 Saint-Denis, France Institut d'Ingénierie de la Santé, UPJV, 3 rue des Louvels, 80000 Amiens, France e-mail:

pallot.adrien@gmail.com

\section{History of Evidence-Based Medicine}

Evidence-Based Medicine (EBM) is an approach that has emerged in medical practice. Over the years, all medical and allied health professions have converged on this new paradigm. The term "medicine" becoming obsolete because of the wide variety of health professions using it, giving way to the term "practice". Sackett already mentioned in 1969 that other health professions could also benefit from EBP enhancements [1]. Evidence-Based Practice (EBP) approach has two main entities: a dimensional triptych and seven steps [2]. The most common definition is that EBP is "the integration of the best research evidence with clinical expertise and patient values" [3] Figure 1. It should be noted that the term "evidence" initially refers to scientific evidence $[4,5]$. Indeed, EBP was born from a desire to change a clinical decision-making paradigm based 


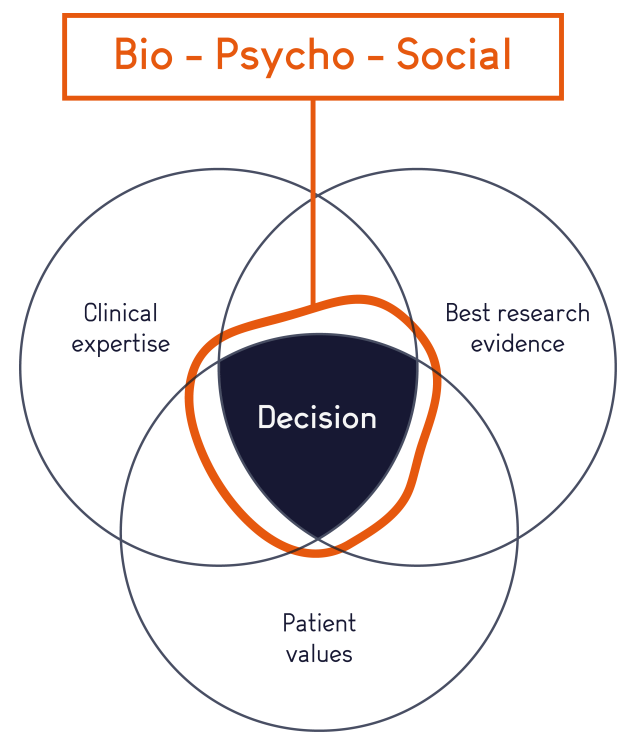

Figure 1 Decision according to the EBP princeps approach

on updated research data because science is in perpetual motion. For that matter, patients and therapists have always been considered in the reasoning to achieve this decision (considering the scientific data alone only becomes critical appraisal of articles). Indeed, the EBP working group declared in 1992 [4] that the "traditional skills of medical training" (pathophysiology, patient listening, therapist behavior,...) should be taken into consideration to "allow the clinician to better judge whether the results of a study are applicable to the patient at hand". This approach is not blindly dictated by scientific data. It is a source of multifactorial weighting. The scientific data, which are in a group referential, provide objective evidence, much less altered by clinical subjectivity. They help answering (or not) to therapeutic questions. Indeed, practice, which is in an individual referential, heals but cannot prove and/or answer certain questions it generates. Figure 2 summarizes this logical and beneficial connection between research and clinical. It's not one or the other: they are thought as a whole.

\section{Purposes of the article}

However, the most widely used definition of EBP does not reflect:

- all the elements to be taken into consideration in this approach to reason

- the notion of relative and weighted reasoned integration of these elements together to generate the choices

- the singularity of a care situation

- the context in which this care situation is

An extended definition would allow a more complete description of these points to better illustrate, in one sentence, the EBP approach. This could also avoid the known pitfalls of its interpretation (EBP is only theory, only scientific studies count, cooking recipes, no autonomy of reasoning, ...). For that reason, the different objectives of this article are:

- to explain why making such a proposal: a new extended definition

- to propose a new extended definition

- to explain our semantic choices

- to propose (and explain) an updated graphic model for the extended definition

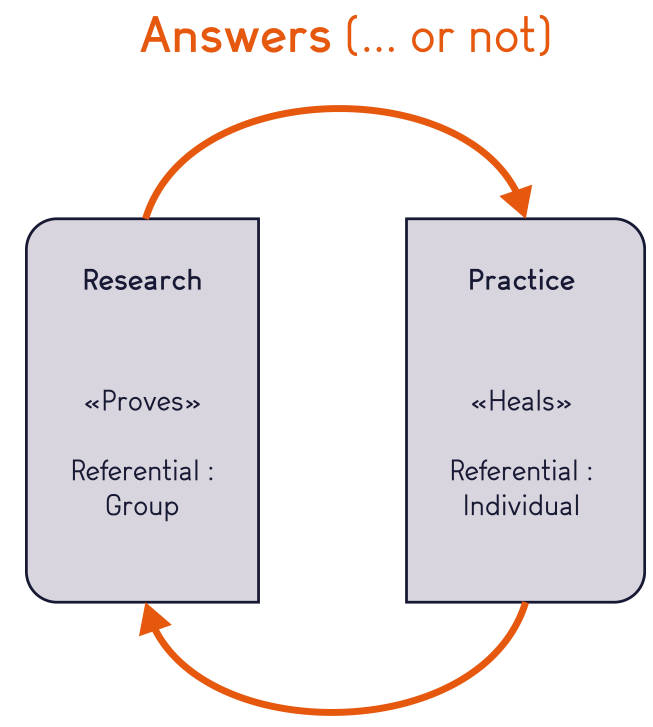

Asks (... or not)

Figure 2 Connection between research and practice

\section{Evolution and additions to the original approach: why proposing a new extended definition}

The care decision resulting from the original EBP approach takes into consideration all three components, in proportions that depend on the care situation Figure 1. Nowadays, this decision must be based on a biopsycho-social approach. Nevertheless, these three dimensions are not exhaustive, other factors may influence these decisions. Rob Herbert et al. mention some of them [6]:

- Cost and/or duration of the intervention

- Therapist's skills

- Regional resources provided for Health

- Cultural influences (including religious)

Other authors have proposed modifications to the original model of EBP. In 2002, Haynes et al. [7] proposed a new model where clinical expertise is the decision-making system (data collection, integration and dissemination):

- Clinician experience is removed from the triptych and replaced by the patient clinical condition and clinical circumstances

- Patients actions are added to their preferences (which may not be similar to their speech, such as a patient wishing to return to physical activity but who actually does not)

- Clinical expertise is placed above the middle of the triptych, straddling the three circles, in a common or exclusive way to a single dimension

As Sarah Wieten comments [8], their corollary is to position clinical expertise as "the force which amalgamates a different set of components together (research evidence, patient preferences and values and the clinical state and circumstances). Rather than a component itself [as it is placed in Figure 1, it is the force that adjudicates between the other components, weighing and balancing the requirements imposed by each component". Nevertheless, we are not convinced by the removal of a clinical aspect in the triptych to place it exclusively on an integrative level. Indeed, this does not allow a correct distinction, as Guy Le Boterf enunciates [9], between "having skills (having resources such as knowledge, know-how, reasoning methods, physical skills, behavioral skills,...) and be competent (be able to act and succeed with relevance 
and competence in a situation of care". Having skills is not enough to act competently.

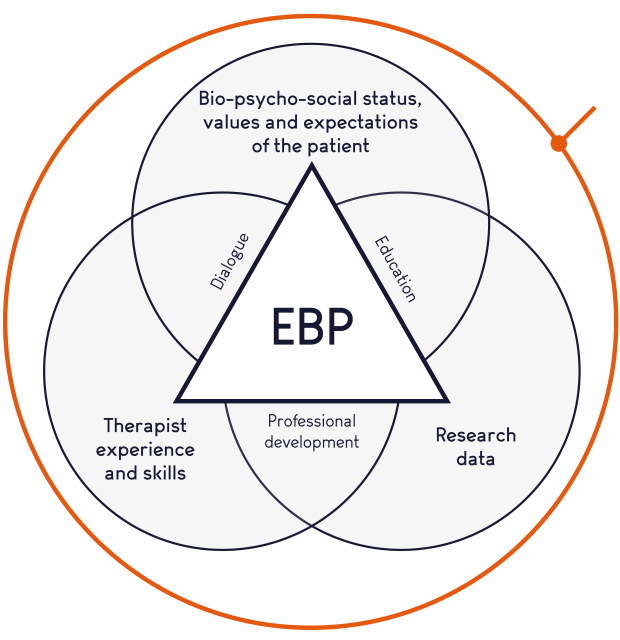

\section{Deontology Public Health Resources Cultures Regulation}

Figure 3 Updated model for the extended definition of an EBP approach

In 2016, Veras et al. [10] add other dimensions such as "ethical principles of autonomy, beneficence, non-maleficence, and justice". They propose a new definition of EBP: it is "an area of study, research, and practice in which clinical decisions are based on the best available evidence, integrating professional practice and expertise with ethical principles". The addition of societal and ethical dimensions is very noteworthy. It places a therapeutic decision in a much larger context: the society in which it is taken. However, concerning the first part of their definition, we prefer to circumscribe EBP as a concept of a practical approach that can be taught, learned, studied and analyzed by research, generating its enhancement; rather than an area integrating these latter aspects, even though one of the original articles was subtitled "a new approach to teaching the practice of medicine" [4]. Indeed, like other care approaches (such as schools of thought that exist in manual therapy), this concept is a way of making a therapeutic decision. This is not a particular type of research.

\section{Proposal for an extended definition [2]}

With its evolution and these successive additions, we wish to propose a new definition of the EBP in order to bring it a more contemporary and complete face: EBP is the reasoned, individualized clinical application of the highest level of evidence possible in a unique multidimensional context of care. Here is the explanation of our semantic choices:

- Application: represents the action of using means (diagnostic, therapeutic, ...) to achieve a concrete goal (in health professions: care and prevention)

- Clinical: refers directly to the practice which is both a starting point (genesis of questioning) and an arrival point (to treat a patient)

- Individualized: this application is adapted to the multidimensional context (which follows in the sentence) based, among other things, on the specificity of each patient. We draw your attention to the fact that we have not used the adjective "individual" because individualized care can be provided within a group session

- Reasoned: refers to the decision-making system aspect (collection, integration and dissemination of practical and theoretical data) of clinical expertise that weights and balances dimensions (between them) to reach a decision

- The highest level of evidence possible: it refers to the hierarchy of evidence mainly based on clinical research. The EBP requires using the best...if we can! The addition of the word "possible" has two meanings: "existing" evidence because there is no evidence on everything (yet! And in this case, we would have to lower our level of requirement and adapt our decision), and "achievable" depending on the multidimensional context (it may not be possible to apply treatment from the best meta-analysis because the patient will not accept it, or we are missing the tools required, ...).

- Multidimensional context of care: the relationship between therapist and patient contains all the dimensions we have mentioned so far (these two people interacting, science, feasibility of interventions, available resources, cultural influences, the healthcare system...), and much more. Table 1 reports the main components with their explanation and content.

- Unique: the equation of all these factors results in reasonably unique therapeutic decisions.

Figure 3 shows the modelling of an EBP decision according to our proposed definition. It presents two referentials: the relationship of care (illustrated by the triptych) and the context in which it takes place (schematized by the circle encompassing the whole). The basic trinomial that we have chosen is the following:

- Bio-psycho-social status, values and expectations of the patient

- Therapist experience and skills

- Research data

It is partially covered by a triangular area that represents clinical expertise, it is the sector where the decision is taken, weighted and balanced between the different dimensions but not necessarily median, as suggested by Haynes et al. [7]. This is where the "being competent" is located, i.e., the implementation of "a relevant professional practice while mobilizing an appropriate combination of resources (other dimensions)" [9]. All around is the dimension of contextual referential in which the care is carried out. It is composed of ethics, public health, available resources, cultures and legislation. Table 1 lists all the dimensions mentioned, as well as their explanation and content.

Finally, we have placed a primordial (but not exclusive) value for each pair of the triptych:

- Dialogue: between therapist and patient, including verbal and nonverbal communication, essential for the acquisition and exchange of information between therapist and patient.

- Education: between the patient and the data of science, it must come from the patient himself (self-education) and by society as a whole (public health messages, learning healthy lifestyle recommendations as early as possible in school, denunciation and fight against fake meds, ...).

- Professional development: between the therapist and scientific data, it is essential to the relevance and safety of care.

This proposal for an extended definition and graphic representation of EBP takes into account the elements of improvement of this model over time and supports its principle of a patient-centered clinical approach. 
Table 1: Explanations and contents of the main dimensions composing an EBP decision

\begin{tabular}{|c|c|c|}
\hline Referentials & Dimensions & Explanations and contents \\
\hline \multirow{4}{*}{ Care relationship } & $\begin{array}{l}\text { Bio-psycho-social } \\
\text { status, values and } \\
\text { expectations of the } \\
\text { patient }\end{array}$ & 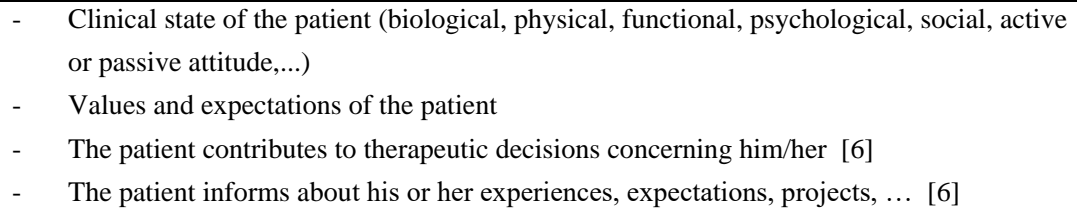 \\
\hline & $\begin{array}{l}\text { Therapist experience } \\
\text { and skills }\end{array}$ & $\begin{array}{l}\text { - The knowledge: } \\
\circ \text { technical } \\
\circ \text { scientific (anatomy, physiology, physiopathology, ...) } \\
\circ \quad \text { contextual in relation to the patient, } \\
\circ \quad \text { organizational, } \\
\circ \quad \text { political, } \\
\circ \quad \ldots\end{array}$ \\
\hline & & $\begin{array}{l}\text { relational: } \\
\text { necessary quality of communication (knowing how to communicate), } \\
\text { empathy (knowing how to put oneself in the place of the other) and } \\
\text { flexibility (knowing how to adapt to the patient's choices and other } \\
\text { dimensions, even if this is not our personal preference) [6] } \\
\text { - } \quad \text { use of cognitive and behavioral techniques [4] }\end{array}$ \\
\hline & Research data & $\begin{array}{ll}\text { - } & \text { From all types of research but more specifically from good quality clinical research [4-6] } \\
\text { - } & \text { Notion of levels of evidence, causality, study designs } \\
\text { - } & \text { Clinically relevant effect size }\end{array}$ \\
\hline \multirow{6}{*}{$\begin{array}{l}\text { Context in which } \\
\text { the care } \\
\text { relationship takes } \\
\text { place }\end{array}$} & $\begin{array}{l}\text { Cost and/or duration } \\
\text { of the intervention }\end{array}$ & $\begin{array}{l}\text { - Consideration of the duration of a treatment (treatment may not be possible due to time } \\
\text { constraints) } \\
\text { - Consideration of the cost of treatment }\end{array}$ \\
\hline & $\begin{array}{l}\text { Regional resources } \\
\text { provided for Health }\end{array}$ & $\begin{array}{l}\text { The resources are uneven in various referentials/frameworks: within the same city, } \\
\text { between the regions of the same country, between the countries in the world, ... }\end{array}$ \\
\hline & $\begin{array}{l}\text { Cultural influences } \\
\text { (including religious) }\end{array}$ & $\begin{array}{l}\text { - Culture in the broad sense can influence expectations, attitudes towards the disease, } \\
\text { therapist-patient interactions, communication, administration of care, .. [6] }\end{array}$ \\
\hline & Public Health & $\begin{array}{ll}- & \text { Policy and actions to improve the population health }\end{array}$ \\
\hline & Deontology & Principles and ethical rules managing and guiding our professional activity \\
\hline & Regulation & $\begin{array}{ll}- & \text { Laws, rules and regulations governing social activities }\end{array}$ \\
\hline
\end{tabular}




\section{Key Points}

- The most widely used definition of EBP does not fully reflect its elements and meanings

- The evolution and additions over time make this approach more complete

- The clinical decision is the result of a balance resulting from the weighting of each dimension between them

- The new extended definition incorporates these points and reflects the singularity of an EBP approach in a care situation

\section{Disclosure of interest}

The authors of this article are the authors of the book from which the manuscript originates.

\section{Ethics}

As this article is an article-based commentary, no ethical approval was required.

\section{Data availability}

As this article is an article-based commentary, no data has been used.

\section{Authors' contribution}

All the authors have reflected and elaborated the new definition (brainstorming sessions). Adrien Pallot wrote the manuscript which was then reviewed by the other authors.

\section{Acknowledgements}

We warmly thank Jonathan Stammers and Beatrice Metharam for their help with accurate English prose and Guillaume Perez for his digital brush.

\section{Fundings}

This research did not receive any specific grant from funding agencies in the public, commercial, or not-for-profit sectors.

\section{References}

[1] David L Sackett. Clinical epidemiology. American journal of epidemiology, 89(2):125-128, 1969.

[2] A Pallot, T Davergne, M Gallois, M Guémann, S Martin, A Morichon, T Osinski, G Raynal, and S Rostagno. Evidence based practice en réducation: démarche pour une pratique raisonnée. Elsevier Health Sciences, 2019.

[3] DL Sackett, SE Strauss, WS Richardson, W Rosengerg, and RB Haynes. Evidence-based medicine: How to practice \& teach EBM. Churchill Livingstone, 2000.

[4] Evidence-Based Medicine Working Group et al. Evidence-based medicine: a new approach to teaching the practice of medicine. Jama, 268:2420-2425, 1992.
[5] David L Sackett, William MC Rosenberg, JA Muir Gray, R Brian Haynes, and W Scott Richardson. Evidence based medicine: what it is and what it isn't, 1996.

[6] Robert Herbert, Gro Jamtvedt, Kåre Birger Hagen, and Judy Mead. Practical evidence-based physiotherapy-E-Book. Elsevier Health Sciences, 2011.

[7] R Brian Haynes, Peter J Devereaux, and Gordon H Guyatt. Clinical expertise in the era of evidence-based medicine and patient choice. BMJ Evidence-Based Medicine, 7(2):36-38, 2002.

[8] Sarah Wieten. Expertise in evidence-based medicine: a tale of three models. Philosophy, ethics, and humanities in medicine, 13(1):1-7, 2018.

[9] Guy Le Boterf. Repenser la compétence. Paris : Eyrolles, 2 edition, 2010.

[10] Mirella Veras, Dahlia Kairy, and Nicole Paquet. What is evidencebased physiotherapy? Physiotherapy Canada, 68(2):95, 2016. 